\title{
ON A CERTAIN DOUBLE INTEGRAL
}

\author{
By A. C. Dixon. \\ [Received January 19th, 1904. - Read February 11th, 1904.]
}

This paper contains a discussion of some properties of the double integral $\int_{0}^{1} \int_{0}^{1} x^{i-1}(1-x)^{j-1} y^{l-1}(1-y)^{k-1}(1-x y)^{m-j-k} d y d x$. The most remarkable of these is that, when multiplied by a certain factor, the expression becomes a symmetric function of five independent variables. From an examination of particular cases it does not seem likely that this function can be reduced to gamma functions in general; though under some conditions it can. (Proc. London Math. Soc., Vol. xxxiv., p. 401; Vol. xxxv., p. 289.)

1. Certain transformations which leave the double integral

$$
\iint x^{i-1}(1-x)^{j-1} y^{l-1}(1-y)^{k-1}(1-x y)^{n-j-k} d x d y
$$

unaltered in form make up a group that is of some interest. Let the transformation

$$
x=\phi(X, Y), \quad y=\psi(X, Y),
$$

where $X, Y$ are the new variables, be denoted by $(\phi(x, y), \psi(x, y))$. Then one member of the group is $(y, x)$, which simply interchanges $x$ and $y$. This operation is of period 2. Others, also of period 2, are $\left(\frac{1}{x}, \frac{1}{y}\right),\left(\frac{1}{x y}, y\right),\left(1-x, \frac{y}{y-1}\right)$. From these may be derived $\left(y, \frac{1}{x y}\right)$, of period 3 , and $\left(\frac{1}{1-x}, \frac{y-1}{y}\right)$, also of period 3 ; and from these again $\left(\frac{1}{1-y}, 1-x y\right)$, which is of period 5 .

The variables introduced in the different transformations are thirty in number, namely,

$$
\begin{aligned}
& x, \frac{1}{x}, \quad 1-x, \frac{1}{1-x}, \frac{x-1}{x}, \frac{x}{x-1} \\
& y, \frac{1}{y}, \quad 1-y, \frac{1}{1-y}, \frac{y-1}{y}, \frac{y}{y-1}
\end{aligned}
$$




$$
\begin{gathered}
x y, \quad \frac{1}{x y}, \quad 1-x y, \frac{1}{1-x y}, \frac{x y-1}{x y}, \frac{x y}{x y-1} ; \\
\frac{1-y}{1-x y}, \frac{1-x y}{1-y}, \frac{y-x y}{1-x y}, \frac{1-x y}{y-x y}, \frac{y-x y}{y-1}, \frac{y-1}{y-x y} ; \\
\frac{1-x}{1-x y}, \frac{1-x y}{1-x}, \frac{x-x y}{1-x y}, \frac{1-x y}{x-x y}, \frac{x-x y}{x-1}, \frac{x-1}{x-x y} .
\end{gathered}
$$

Let $P, Q, R, S, T$ denote these five rows respectively. Then the transformations of the group permute $P, Q, R, S, T$. For instance, $(y, x)$ interchanges $P$ and $Q$, also $S$ and $T$. We may then write

and similarly

$$
(y, x)=(P Q)(S T),
$$

$$
\left(\frac{1}{x}, \frac{1}{y}\right)=(S T), \quad\left(\frac{1}{x y}, y\right)=(P R)(S T), \quad\left(1-x, \frac{y}{y-1}\right)=(R S) .
$$

From these four all the permutations may be derived. Conversely: each permutation corresponds only to one operation of the group; for, if not, an operation of the group, say $\left(x^{\prime}, y^{\prime}\right)$, other than $(x, y)$ would leave $P, Q, R, S, T$ unchanged.

Now, since $P$ is unchanged,

$$
x^{\prime}=x, \frac{1}{x}, 1-x, \frac{1}{1-x}, \frac{x}{x-1}, \text { or } \frac{x-1}{x} .
$$

Since $Q$ is unchanged,

$$
y^{\prime}=y, \frac{1}{y}, 1-y, \frac{1}{1-y}, \frac{y}{y-1}, \text { or } \frac{y-1}{y} .
$$

Since $R$ is unchanged, $x^{\prime} y^{\prime}$ is a function of $x y$ only, which can only be if $x^{\prime}=x, y^{\prime}=y$ or $x^{\prime}=x^{-1}, y^{\prime}=y^{-1}$. The latter is out of the question, since it interchanges $S$ and $T$. Hence the group of transformations is the same in structure as that of the permutations of five letters.

This may be seen in another way. Put

$$
x=\frac{(a-b)(c-d)}{(c-b)(a-d)}, \quad y=\frac{(a-e)(c-b)}{(c-e)(a-b)} .
$$

Then the operations of the group are equivalent to permutations of $a, b$, 
$c, d, e$. We have, in fact,

$$
\begin{aligned}
(a c) & =\left(\frac{1}{x}, \frac{1}{y}\right), & (d e) & =\left(\frac{1}{y}, \frac{1}{x}\right), \\
(b d) & =\left(\frac{1}{x}, x y\right), & (b c) & =\left(1-x, \frac{y}{y-1}\right), \\
(a c)(d e) & =(y, x), & (a c)(b e) & =\left(\frac{1}{x y}, y\right),
\end{aligned}
$$

and $P, Q, R, S, T$ are replaced by $e, d, b, c, a$ respectively. Let us slightly modify the mesning of $P, Q, R, S, T$ and take

$$
P=\frac{\left(1-x+x^{2}\right)^{3}}{\left(x-x^{2}\right)^{2}},
$$

$Q, R, S, T$ the same functions of $y, x y, \frac{1-y}{1-x y}, \frac{1-x}{1-x y}$ that $P$ is of $x$. Then $P$ is a symmetric function of $a, b, c, d ; Q$ the same of $a, b, c, e$; and so on ; and, if we suppose $a, b, c, d, e$ the roots of a quintic equation, $P, Q, R, S, T$ will be those of a resolvent of the same degree whose coefficients are all invariants: this resolvent could be derived by a Tschirnhausen transformation.

It is easily found that the leading coefficient in this resolvent is the cube of the discriminant, and that the second and third coefficients contain the second and first powers of the discriminant as factors respectively. Each coefficient is of the order 24 in those of the original quintic. The discriminant of the resolvent contains as factors the seventh power of the original discriminant and the fourth power of the skew invariant, since this latter vanishes when two pairs of roots belong to an involution in which the fifth root is self-conjugate. If $a b, c d$ are the two psirs, we have $(a b c e)=(b a d e),(a c d e)=(b d c e)$; so that the resolvent has two pairs of equal roots.

Since the discriminant is of order 8 , the skew invariant of order 18 , and the discriminant of the resolvent of order 192, the last must contain another factor of order 64 , which is found to be the square of an invariant of order 32 whose vanishing is the condition that four roots, such as $a, b$, $c, d$, should be homographic with $b, c, a, e .^{*}$

- Note added March 15th, 1904.-The group of permutations of five letters is here shown to be isomorphic with a group of birational transformations of two variables. This is a case of the theorem, given by Prof. W. Burnside and Prof. E. H. Moore (see Messenger of Mathematics, February, 1901, pp. 148-153 ; and American Journal, Vol. xxIr., pp. 279-291), that the symmetric group of degree $n$ can be represented as a group of birational trunsformations in $n-3$ symbols. 
2. Each transformation of the group produces a linear transformation of the letters $i, j, k, l, m$ which occur in the double integral.

Thus $i, j, k, l, m$ are changed

by $(y, x)$ into $l, k, j, i, m$;

by $\left(\frac{1}{x}, \frac{1}{y}\right)$ into $k-m-i+1, j, k, j-m-l+1, m$;

by $\left(\frac{1}{x y}, y\right)$ into $k-m-i+1, m-j-k+1, k, l-i-j+1, m$;

by $\left(1-x, \frac{y}{y-1}\right)$ into $j, i, j-m-l+1, l, i-k-l+1$.

Constant factors of the subject of integration are here neglected.

These transformations permute the ten quantities $i, j, k, l, m$, $i-k-l+1, \quad j-l-m+1, \quad k-m-i+1, \quad l-i-j+1, \quad m-j-k+1 \quad$ in such a way that rows and columns are permuted simultaneously in the array :-

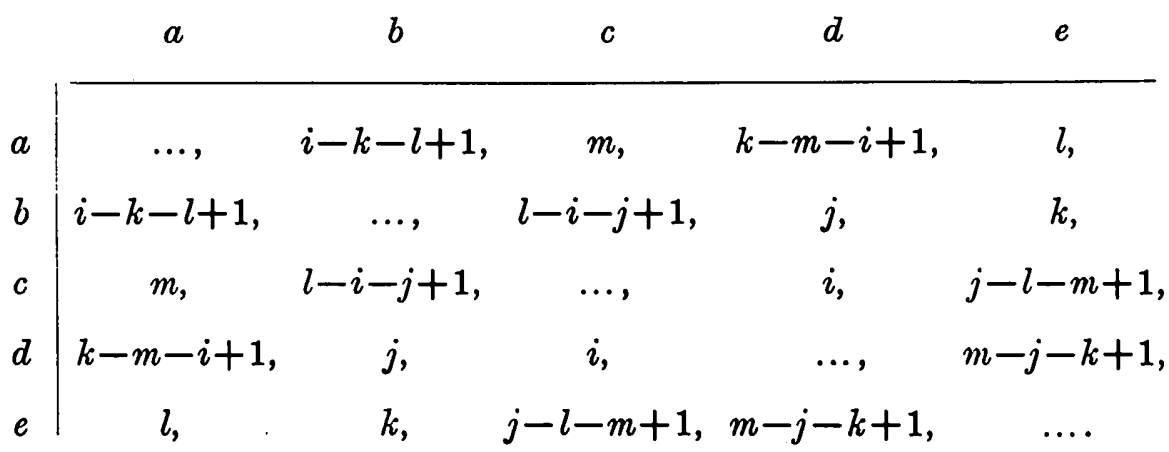

The array is bordered with the letters $a, b, c, d, e$ to show the correspondence between the two forms of the group.

2. So far the double integral has been taken as indefinite. If we now make it definite, the limits being 0,1 for both $x$ and $y$, there are ten transformations of the group that leave the limits unchanged. One of these is $(y, x)$; another is $\left(\frac{1-x}{1-x y}, y\right)$ or $(a e)(b c)$, which substitutes $j, i, m, l, k$ for $i, j, k, l, m$. These operations reverse the cyclic order of $i, j, k, l, m$, as also of $a, b, c, e, d$; hence the ten operations of the 
sub-group are those which maintain or reverse the cyclic order $i, j, k, l, m$, and the value of

$$
\int_{0}^{1} \int_{0}^{1} x^{i-1}(1-x)^{j-1} y^{l-1}(1-y)^{k-1}(1-x y)^{m-j-k} d x d y
$$

is unchanged by these ten substitutions for $i, j, k, l, m$.

Denote this integral by $B(i, j, k, l, m)$ on the supposition that the real parts of $i, j, k, l, m$ are positive, so that the value is finite. It can be expressed as a single integral by means of the hypergeometric function thus

$$
\frac{\Gamma k \Gamma l}{\Gamma(k+l)} \int_{0}^{1} x^{i-1}(1-x)^{j-1} F(j+k-m, l, k+l, x) d x .
$$

Since the hypergeometric function is unaffected by an interchange of its first two elements, we find that $B(i, j, k, l, m) \div \Gamma k \Gamma l$ is unchanged by the substitution for $i, j, k, l, m$ of $i, j, l+m-j, j+k-m, m$. Since $i, j, m$ are also unchanged, we have $B(i, j, k, l, m) \div \Gamma i \Gamma j \Gamma k \Gamma l \Gamma m$ unaffected by the same substitution, which replaces $i+j, j+k, k+l, l+m$, $m+i$ by $i+j, l+m, k+l, j+k, m+i$, the same quantities in another cyclic order.

Since it has been proved already that $B(i, j, k, l, m)$ is unaffected by any reversal of the cyclic order of the five letters, we find that

$$
B(i, j, k, l, m) \div \Gamma i \Gamma j \Gamma k \Gamma l \Gamma m
$$

is a symmetric function of $i+j, j+k, k+l, l+m, m+i$.

Writing $F(\alpha, \beta, \gamma, \delta, \epsilon, x)$ for

$$
1+\frac{\alpha \beta \gamma}{\delta \epsilon} x+\frac{\alpha(\alpha+1) \beta(\beta+1) \gamma(\gamma+1)}{1.2 . \delta(\delta+1) \epsilon(\epsilon+1)} x^{2}+\ldots,
$$

we have, when the real parts of $\delta+\epsilon-a-\beta-\gamma, a, \beta, \delta-a, \epsilon-\beta$ are positive,

$\Gamma \alpha \Gamma(\delta-\alpha) \Gamma \beta \Gamma(\epsilon-\beta) F(\alpha, \beta, \gamma, \delta, \epsilon, 1)$

$$
=\Gamma \delta \Gamma \epsilon \int_{0}^{1} \int_{0}^{1} x^{\alpha-1}(1-x)^{\delta-\alpha-1} y^{\beta-1}(1-y)^{e-\beta-1}(1-x y)^{-\gamma} d x d y .
$$

Hence

$$
F(a, \beta, \gamma, \delta, \epsilon, 1) \div \Gamma \delta \Gamma_{\epsilon} \Gamma(\delta+\epsilon-\alpha-\beta-\gamma)
$$

is a symmetric function of $\delta, \epsilon, \delta+\epsilon-\alpha-\beta, \delta+\epsilon-\beta-\gamma, \delta+\epsilon-\gamma-\alpha$.

Write $p, q, r, s, t$ for these five variables; then when $\alpha=0$, that 
is, when $p+q+s=r+t$, the value of the symmetric function of $p, q, r, s, t$ is $1 / \Gamma p \Gamma q \Gamma s$. Another interesting result is that

$$
\begin{aligned}
& \frac{F(\alpha, \beta, \gamma, \delta, \epsilon, 1)}{\Gamma \delta \Gamma \epsilon(\delta+\epsilon-\alpha-\beta-\gamma)} \\
& \quad=\frac{F(\delta+\epsilon-\alpha-\beta-\gamma, \epsilon-\alpha, \delta-\alpha, \delta+\epsilon-\alpha-\beta, \delta+\epsilon-\alpha-\gamma, 1)}{\Gamma(\delta+\epsilon-\alpha-\beta) \Gamma(\delta+\epsilon-\alpha-\gamma) \Gamma \alpha},
\end{aligned}
$$

from which it follows that

$$
\lim _{a+\beta+\gamma=\delta+e} \frac{F(\alpha, \beta, \gamma, \delta, \epsilon, 1)}{\Gamma(\delta+\epsilon-\alpha-\beta-\gamma)}=\frac{\Gamma \delta \Gamma_{\epsilon}}{\Gamma \alpha \Gamma \beta \Gamma \gamma} .
$$

4. The symmetry of the hypergeometric function $F(\alpha, \beta \gamma, t)$ with respect to $\alpha, \beta$ may be shown by transformation of the definite integral as follows. We have

$$
\begin{aligned}
\int_{0}^{1} \int_{0}^{1} x^{\beta-1}(1-x)^{\gamma-\beta-1} y^{\alpha-1}(1-y)^{\gamma-a-1} & (1-x t)^{-a} d x d y \\
& =B(\beta, \gamma-\beta) B(\alpha, \gamma-\alpha) F(\alpha, \beta, \gamma, t) .
\end{aligned}
$$

Put

$$
\frac{x}{1-x}=\frac{u}{(1-u)(1-v t)}, \quad \frac{y}{(1-y)(1-x t)}=\frac{v}{1-v} \text {. }
$$

This gives

$$
\begin{aligned}
& x=\frac{u}{1-v t+u v t}, \quad y=\frac{v(1-u t-v t+u v t)}{1-v t} \\
& (1-x)(1-y)=(1-u)(1-v), \quad \frac{\partial(x, y)}{\partial(u, v)}=\frac{x y}{u v},
\end{aligned}
$$

and the double integral becomes

$$
\int_{0}^{1} \int_{0}^{1} u^{\beta-1}(1-u)^{\gamma-\beta-1} v^{u-1}(1-v)^{\gamma-a-1}(1-v t)^{-\beta} d u d v,
$$

the same expression with $\alpha, \beta$ interchanged.

5. It would seem that there is no irreducible closed path which for all values of the indices $i, j, k, l, m$ brings back the expression

$$
x^{i-1}(1-x)^{j-1} F\left(j+k^{-m}, l, k+l, x\right)
$$

to its original value. Any closed path causes a linear homogeneous transformation of $F$ and another function $F_{1}$; if the values of $x^{i-1}$, $(1-x)^{j-1}$ are to be restored in general, the path must pass round each of the points 0,1 as often positively as negatively, and the product of the multipliers in the transformation of $F, F_{1}$ must therefore be 1 . If $F$, or any expression of the form $\lambda F+\mu F_{1}$, is unchanged, one of the multipliers 
is 1 , the other is then also 1 , and the transformation is parabolic or else identical. But there are well known cases in which the group of the hypergeometric function contains no parabolic substitutions. Such cases are, for instance, those associated with the regular solids. When the first three elements are commensurable there are irreducible paths corresponding to identical substitutions, but these are not the same for all values of the elements.

6. The function $B(i, j, k, l, m)$ satisfies certain difference equations which may be found as follows :-

Multiply the identity $x+(1-x)=1$ by

$$
x^{i-1}(1-x)^{j-1} y^{l-1}(1-y)^{k-1}(1-x y)^{m-j-k}
$$

and integrate.

Thus $B(i+)+B(j+, m+)=B$, where $i+$ indicates that $i$ is to be increased by 1 , the other letters being unchanged.

Other equations may be derived from this by symmetry. Write $p, q, r, s, t$ for $i+j, j+k, k+l, l+m, m+i$, and $C(p, q, r, s, t)$ or simply $C$ for $B(i, j, k, l, m) / \Gamma i \Gamma j \mathrm{I} k \Gamma l \Gamma m$. Thus we have

$$
\begin{aligned}
C= & i C(p+, t+)+j m C(p+, q+, s+, t+) \\
= & \frac{1}{2}(p-q+r-s+t) C(p+, t+) \\
& \quad+\frac{1}{4}(p+q-r+s-t)(-p+q-r+s+t) C(p+, q+, s+, t+) .
\end{aligned}
$$

Of such equations there are thirty, since $p, q, r, s, t$ may be interchanged in any way. If we take the one written with those derived from it by interchanging $q, s, t$, we can eliminate $C$ and $C(p+, q+, s+, t+)$. Thus

$$
\begin{aligned}
(p-q+r-s+t)(q-s) C(p+, t+) & +(p+q+r-s-t)(s-t) C(p+, q+) \\
& +(p-q+r+s-t)(t-q) C(p+, s+)=0,
\end{aligned}
$$

or, by the substitution of $p-1$ for $p$,

$$
\begin{aligned}
(p-q+r-s+t-1)(q-s) C(t+) & +(p+q+r-s-t-1)(s-t) C(q+) \\
+ & (p-q+r+s-t-1)(t-q) C(s+)=0 .
\end{aligned}
$$

Similarly, interchanging $q, r, s$, we can form from (a) two more equations by means of which it is possible to eliminate $C$ and $C(p+, t+)$ : then, by diminishing $p, q, r, s, t$ each by 1 , we have

$$
\begin{aligned}
& (q-s)(p+q-r+s-t-1)(-p+q-r+s+t-1) C(r-) \\
& \quad+(s-r)(p-q+r+s-t-1)(-p-q+r+s+t-1) C(q-) \\
& \quad+(r-q)(p+q+r-s-t-1)(-p+q+r-s+t-1) C(s-)=0 .
\end{aligned}
$$


Again, from (a), by interchanging $q, t$ and eliminating

$$
C(p+, q+, s+, t+),
$$

we have

$4(q-t) C=(p+q+r-s-t)(p+q-r+s-t) C(p+, q+)$

$$
-(p-q+r-s+t)(p-q-r+s+t) C(p+, t+) ;
$$

this may be written

$$
(i-j-k+m) B=(i-k+m) B(i+)-(j+k-m) B(j+),
$$

in which form it seems to correspond with the reduction formula

$$
(m+n) B(m, n+1)=n B(m, n) .
$$

To get some of these results we might also have integrated by parts.

Froin the equation (a) form two more, (1) by interchanging the pair $p t$ with $q s$, (2) by writing $p-1, t-1$ for $p, t$. From these three eliminate $C(q+, s+)$ and $C(p+, q+, s+, t+)$. The result contains $C(p-, t-), C$, and $C(p+, t+)$, and may therefore be written as a difference equation of the second order for $B$ or $C$, with the single independent variable $i$, the other four $j, k, l, m$ being treated as constant. This equation is

$$
\begin{array}{r}
(i+m-k)(i+j-l) B(i+)+\{(i-1)(k+l-i)+j m-(i+j-l)(i+m-k) ; B \\
-(i-1)(k+l-i) B(i-)=0 .
\end{array}
$$

Its general solution is discussed in Boole's Finite Differences, pp. 260-2 (1880). 